\title{
Euclidean homotopic skeleton based on critical kernels
}

\author{
M. Couprie ${ }^{(1)}$, A. V. Saúde ${ }^{(1,2)}$, G. Bertrand ${ }^{(1)}$ \\ (1) Institut Gaspard-Monge, Laboratoire A2SI, Groupe ESIEE \\ BP99, 93162 Noisy-le-Grand Cedex France \\ (2) State University of Campinas, School of Electrical and Computer Engineering \\ DCA-FEEC-UNICAMP, Caixa Postal 6101, 13081-970 Campinas/SP, Brazil
}

\begin{abstract}
Critical kernels constitute a general framework settled in the category of abstract complexes for the study of parallel thinning in any dimension. It allows to easily design parallel thinning algorithms which produce new types of skeletons, with specific geometrical properties, while guaranteeing their topological soundness. In this paper, we demonstrate that it is possible to define a skeleton based on the Euclidean distance, rather than on the common discrete distances, in the context of critical kernels. We provide the necessary definitions as well as an efficient algorithm to compute this skeleton.
\end{abstract}

Key Words: parallel thinning, Euclidean distance, medial axis, homotopy, critical kernels

\section{Introduction}

In 1961, H. Blum [4] introduced the notion of medial axis or skeleton, which has since been the subject of numerous theoretical studies and has also proved its usefulness in practical applications. Although originally defined in the continuous plane using a "grassfire" analogy, the medial axis can also be defined using the notion of maximal inscribed ball, in $n$-dimensional continuous or discrete spaces $[5,10]$. The medial axis is a set of points which is, by nature, centered in the object with respect to the distance which is used to define the notion of ball. In the discrete $n$ dimensional grid $\mathbb{Z}^{n}$, in order to achieve a certain degree of rotation invariance, the Euclidean distance between points of $\mathbb{Z}^{n}$ may be used. Nevertheless, even in this case, the medial axis has not, in general, the same nice properties as its continuous counterpart. In particular, the properties of thinness and homotopy (or topology preservation) are lost.

In order to obtain an homotopic skeleton which contains the medial axis, the use of guided and constrained discrete homotopic transformations has been proposed by several authors (see e.g. [7, 13, 12, 6]). Intuitively, the principle of these methods is to sequentially delete simple points (that is, points which can be deleted without changing topology) from the object, taking in first priority the points which are closest to the background, and preserving the medial axis points. These approaches suffer from two drawbacks. First, the result of the method is not uniquely defined: depending on the order in which points with equal priority are processed, different results can be obtained. Second, the obtained skeleton is not thin since it contains the medial axis which can be "thick" at some places. Let us mention that some authors considered higher resolution grids in order to get thinner skeletons (see e.g. [1, 8]), but none of these works was based on the Euclidean distance.

Recently, G. Bertrand introduced a general framework for the study of parallel thinning in any dimension in the context of abstract complexes [2]. A new definition of a simple point is proposed in [2], based on the collapse operation which is a classical tool in algebraic topology and which guarantees topology preservation. Then, the notions of an essential face and of a core of a face allow to define the critical kernel $\mathcal{K}$ of an object $X$. The most fundamental result proved in [2] is that, if a subset $Y$ of $X$ contains $\mathcal{K}$, then $X$ collapses onto $Y$, i.e., $Y$ is a retraction of $X$.

In [3], the particular case of 2D structures in spaces of two and three dimensions is considered. Several new parallel thinning algorithms are proposed and compared with the existing ones, when possible. For example, one of these new algorithms called $A K^{2}$ is proved to preserve the medial axis (defined with the so-called 4-distance) and is invariant with respect to $\pi / 2$ rotations, another one called $N K^{2}$ produces a result which is proved to contain the medial axis and to be minimal for this property. These algorithms have no equivalent in the literature. Furthermore, the results of the algorithms proposed in [3] are well-defined, they do not depend on any arbitrary choice.

In Fig. 1, we motivate the need for a skeleton based on the Euclidean distance. First, let us notice that the balls for the 4-distance are squares with sides rotated by $\pi / 4$ with respect to the main axes (like the rightmost square in Fig. 1). 
This explains why the skeleton based on this distance is not invariant by a rotation of $\pi / 4$ (see figure). Also, due to the particular shape of these balls, diagonal branches of the skeleton appear, even when they correspond to parts of the contour which are not very salient (see A, B, C, D), whereas more salient features (see $\mathrm{X}, \mathrm{Y}$ ) do not generate any skeleton branch.

The goal of this paper is to provide a definition and an algorithm for such an Euclidean skeleton. This skeleton should contain the Euclidean medial axis of the original object, thus we also propose a definition of the Euclidean medial axis in the context of cubical complexes embedded in $\mathbb{Z}^{n}$. This medial axis can be efficiently computed thanks to an algorithm recently introduced in [11].

Let us emphasize the fact that the same approach is also valid in 3D. An extended version of this paper will provide definitions, algorithms and results in both $2 \mathrm{D}$ and $3 \mathrm{D}$ spaces.

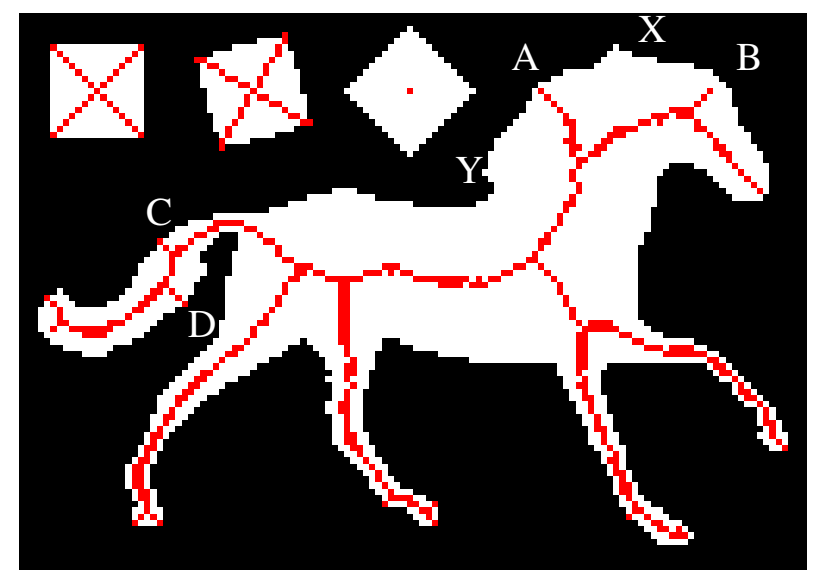

Figure 1. Skeleton obtained by the parallel algorithm $A K^{2}$ [3], this skeleton contains the (4-distance) medial axis of the shape.

\section{Basic notions}

We recall briefly in sections 1 and 2, the definitions and results from $[2,3]$ which are used in the sequel.

Let $\mathbb{Z}$ be the set of integers. We consider the families of sets $\mathbb{F}_{0}^{1}, \mathbb{F}_{1}^{1}$, such that $\mathbb{F}_{0}^{1}=\{\{a\} \mid a \in \mathbb{Z}\}, \mathbb{F}_{1}^{1}=\{\{a, a+$ 1\} $\mid a \in \mathbb{Z}\}$. A subset $f$ of $\mathbb{Z}^{n}, n \geq 2$, which is the Cartesian product of exactly $m$ elements of $\mathbb{F}_{1}^{1}$ and $(n-m)$ elements of $\mathbb{F}_{0}^{1}$ is called a face or an $m$-face of $\mathbb{Z}^{n}, m$ is the dimension of $f$, we write $\operatorname{dim}(f)=m$.

We denote by $\mathbb{F}^{2}$ the set composed of all $m$-faces of $\mathbb{Z}^{2}$, $m=0,1,2$. An $m$-face of $\mathbb{Z}^{2}$ is called a point if $m=0$, a (unit) interval if $m=1$, a (unit) square if $m=2$.
Let $f$ be a face in $\mathbb{F}^{2}$. We set $\hat{f}=\left\{g \in \mathbb{F}^{2} \mid g \subseteq f\right\}$ and $\hat{f}^{*}=\hat{f} \backslash\{f\}$. Any $g \in \hat{f}$ is a face of $f$, and any $g \in \hat{f}^{*}$ is a proper face of $f$. If $X$ is a finite set of faces of $\mathbb{F}^{2}$, we write $X^{-}=\cup\{\hat{f} \mid f \in X\}, X^{-}$is the closure of $X$.

A set $X$ of faces of $\mathbb{F}^{2}$ is a cell or an $m$-cell if there exists an $m$-face $f \in X$, such that $X=\hat{f}$. The boundary of a cell $\hat{f}$ is the set $\hat{f}^{*}$.

A finite set $X$ of faces of $\mathbb{F}^{2}$ is a complex (in $\mathbb{F}^{2}$ ) if for any $f \in X$, we have $\hat{f} \subseteq X$. Any subset $Y$ of a complex $X$ which is also a complex is a subcomplex of $X$. If $Y$ is a subcomplex of $X$, we write $Y \preceq X$.

If $X$ is a complex in $\mathbb{F}^{2}$, we also write $X \preceq \mathbb{F}^{2}$.

A face $f \in X$ is principal for $X$ if there is no $g \in X$ such that $f \in \hat{g}^{*}$. We denote by $X^{+}$the set composed of all principal faces of $X$. Observe that $\left[X^{+}\right]^{-}=X^{-}$and thus, that $\left[X^{+}\right]^{-}=X$ whenever $X$ is a complex. In Fig. 2, we give some illustrations of the notions defined above.

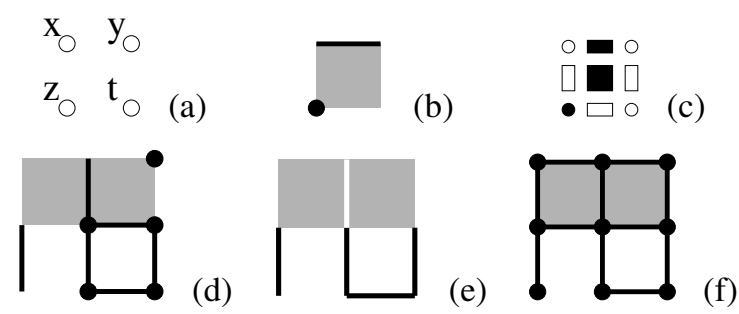

Figure 2. (a): Four points $x, y, z, t$ of $\mathbb{Z}^{2}$ such that $\{x, y, z, t\}$ is a 2-face. (b,c): Two representations of the set of faces $\{\{x, y, z, t\},\{x, y\},\{z\}\} . \quad$ (d): A set $X$ of faces in $\mathbb{F}^{2}$, we see that $X$ is not a complex. (e): The set $X^{+}$, composed by the principal faces of $X$. (f): The set $X^{-}$, i.e. the closure of $X$, which is a complex.

Let $X$ be a complex in $\mathbb{F}^{2}$ and let $f \in X^{+}$. The face $f$ is a border face for $X$ if there exists one face $g \in \hat{f}^{*}$ such that $f$ is the only face of $X$ which contains $g$. Such a face $g$ is said to be free for $X$ and the pair $(f, g)$ is said to be a free pair for $X$. We say that $f \in X^{+}$is an interior face for $X$ if $f$ is not a border face.

Let $X$ be a complex, and let $(f, g)$ be a free pair for $X$. The complex $X \backslash\{f, g\}$ is an elementary collapse of $X$.

Let $X, Y$ be two complexes. We say that $X$ collapses onto $Y$ if there exists a collapse sequence from $X$ to $Y$, i.e., a sequence of complexes $\left\langle X_{0}, \ldots, X_{l}\right\rangle$ such that $X_{0}=X$, $X_{l}=Y$, and $X_{i}$ is an elementary collapse of $X_{i-1}, i=$ $1, \ldots, l$. If $X$ collapses onto $Y$, we also say that $Y$ is a retraction of $X$. 


\section{Critical kernels}

Let us briefly recall (for the 2D case) the framework introduced by G. Bertrand in [2] for thinning, in parallel, discrete objects with the warranty that we do not alter the topology of these objects. This method is based solely on three notions, the notion of an essential face which allows to define the core of a face, and the notion of a critical face. Definition 1. Let $X \preceq \mathbb{F}^{2}$ and let $f \in X$. We say that $f$ is an essential face for $X$ if $f$ is precisely the intersection of all principal faces of $X$ which contain $f$, i.e., if $f=\cap\{g \in$ $\left.X^{+} \mid f \subseteq g\right\}$. We denote by $\operatorname{Ess}(X)$ the set composed of all essential faces of $X$. If $f$ is an essential face for $X$, we say that $\hat{f}$ is an essential cell for $X$.

Observe that a principal face for $X$ is necessarily an essential face for $X$, i.e., $X^{+} \subseteq \operatorname{Ess}(X)$.

In the following, $K$ will denote a subset of $X$ which plays the role of a constraint set, that is, a set of faces which must be preserved during the thinning.

Definition 2. Let $X \preceq \mathbb{F}^{2}$, let $K \subseteq X$ and let $f \in \operatorname{Ess}(X)$. The core of $\hat{f}$ for $\langle X, K\rangle$ is the complex Core $(\hat{f}, X, K)=$ $\cup\left\{\hat{g} \mid g \in\left[K^{-} \cup \operatorname{Ess}(X)\right] \cap \hat{f}^{*}\right\}$.

Definition 3. Let $X \preceq \mathbb{F}^{2}$, let $K \subseteq X$ and let $f \in X$. We say that $f$ and $\hat{f}$ are regular for $\langle X, K\rangle$ if $f \in E s s(X)$ and if $\hat{f}$ collapses onto Core $(\hat{f}, X, K)$. We say that $f$ and $\hat{f}$ are critical for $\langle X, K\rangle$ if $f \in E s s(X)$ and if $f$ is not regular for $\langle X, K\rangle$.

If $X \preceq \mathbb{F}^{2}$, we set Critic $(X, K)=\cup\{\hat{f} \mid f$ is critical for $\langle X, K\rangle\}$, Critic $(X, K)$ is the critical kernel of $X$ constrained by $K$, and $C r i t i c(X, \emptyset)$ is the critical kernel of $X$.

In Fig. 3a, the letters $A, B, C, D, E$ indicate 2faces, the letters $a, b, c, d, e, f 1$-faces, and the letters $t, u, v, w, x, y, z 0$-faces. The essential faces of the complex $X$ are highlighted: we have $\operatorname{Ess}(X)=$ $\{A, B, C, D, E, a, b, c, x, y, z\}$. The core of $\hat{C}$ for $\langle X, \emptyset\rangle$ is the set $\{x, b, y, z\}$, the core of $\hat{D}$ for $\langle X, \emptyset\rangle$ is $\{y, c, u\}$. We can see that a collapse sequence from $\hat{D}$ to $\{y, c, u\}$ exists: e.g., $\langle\hat{D},\{v, e, w, f, y, c, u\},\{w, f, y, c, u\},\{y, c, u\}\rangle$. Thus, the face $D$ is regular for $\langle X, \emptyset\rangle$. On the other hand, it can be seen that no collapse sequence from $\hat{C}$ to $\{x, b, y, z\}$ exists: the face $C$ is critical for $\langle X, \emptyset\rangle$. Let $K=\{v\}$, the core of $\hat{D}$ for $\langle X, K\rangle$ is $\{y, c, u, v\}$, and we can see that $D$ is critical for $\langle X, K\rangle$. Fig. 3b shows the critical kernel of $X$, and Fig. 3c shows the critical kernel of $X$ constrained by $K$.

The following theorem holds in arbitrary dimension.

Theorem 4 ([2]). Let $X \preceq \mathbb{F}^{2}$, let $K \subseteq X$. The critical kernel of $X$ constrained by $K$ is a retraction of $X$.

\section{Euclidean medial axis in $\mathbb{F}^{2}$}

The definition of the medial axis in terms of centers of maximal balls [5] can be used in any metric space, e.g.

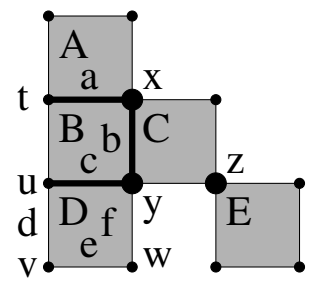

(a)

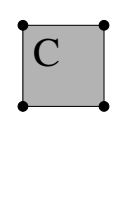

(b)

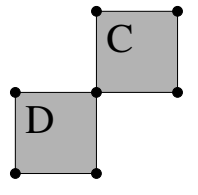

(c)
Figure 3. (a): A complex $X$ for the illustration of the notions of essential face, core and critical face (see text). (b): The critical kernel $\operatorname{Critic}(X, \emptyset)$. (c): The constrained critical kernel $\operatorname{Critic}(X, K)$, with $K=\{v\}$.

a subset $G$ of $\mathbb{Z}^{n}$ (a grid) equipped with a distance. Let $G$ be a subset of $\mathbb{Z}^{n}$, let $x$ and $y$ be two points of $G$, we denote by $d(x, y)$ the Euclidean distance between $x$ and $y$. For defining and computing the medial axis, it is in fact sufficient to consider the squared Euclidean distance $d^{2}(x, y)=\sum_{i=1}^{n}\left(x_{i}-y_{i}\right)^{2}$.

In most works on discrete medial axis, the whole discrete grid $\mathbb{Z}^{n}$ is taken for $G$. It means that both object points and centers of balls must belong to the same set. In the following, we will consider families of balls which are made of points of $G$ but not necessarily centered on points of $G$, where $G$ is a subsampling of $\mathbb{Z}^{n}$. This motivates some of the definitions given in this section, which are slightly different from the traditional ones.

Let $R \in \mathbb{N}, x \in G$, the (Euclidean) ball in $G$ centered on $x$ with (squared) radius $R$ is defined by $B(x, R)=$ $\left\{y \in G \mid d^{2}(x, y) \leq R\right\}$. Let $X \subseteq G, x \in X$, we denote by $D_{X}(x)$ and by $D_{X}^{2}(x)$ the Euclidean distance and the squared Euclidean distance, respectively, from $x$ to the nearest point $y \in G \backslash X$. We say that $D_{X}$ (resp. $D_{X}^{2}$ ) is the distance map (resp. squared distance map) of $X$.

Let $X$ be any subset of $G$, let $x \in X$, we say that a ball $B=B(x, R)$ in $G$ is maximal for $X$ (in $G$ ) if $B$ is included in $X$ and maximal for this property, more precisely if $B \subseteq B\left(y, R^{\prime}\right) \subseteq X$, with $y \in G$ and $B\left(y, R^{\prime}\right)$ being a ball in $G$, implies $B\left(y, R^{\prime}\right)=B$. The (Euclidean) medial axis of $X$ (in $G$ ), denoted by $\mathrm{MA}(X, G)$ or simply $\mathrm{MA}(X)$ when no confusion may occur, is the set of centers of all maximal Euclidean balls for $X$ in $G$.

In order to define an Euclidean medial axis in $\mathbb{F}^{2}$, we have to define a distance in such a space, as close as possible to the Euclidean distance. The easiest way to do so is to take advantage of a "natural" bijection between $\mathbb{F}^{2}$ and $\mathbb{Z}^{2}$, defined below.

Definition 5. Let $f \in \mathbb{F}^{2}$, let $a, b \in \mathbb{Z}^{2}$. We define the bijection $\Psi$ from $\mathbb{F}^{2}$ to $\mathbb{Z}^{2}$, as follows:

if $f=\{(a, b),(a, b+1),(a+1, b),(a+1, b+1)\}($ a 2-face $)$, then $\Psi(f)=(2 a, 2 b)$; 
if $f=\{(a, b),(a, b+1)\}$ (a vertical 1-face),

then $\Psi(f)=(2 a-1,2 b)$;

if $f=\{(a, b),(a+1, b)\}$ (an horizontal 1-face),

then $\Psi(f)=(2 a, 2 b-1)$;

if $f=\{(a, b)\}($ a 0 -face $)$,

then $\Psi(f)=(2 a-1,2 b-1)$.

Let $F \preceq \mathbb{F}^{2}$, we define the embedding of $F$ in $\mathbb{Z}^{2}$ as $\Psi(F)=\{\Psi(f) \mid f \in F\}$.

This correspondence is illustrated in Fig. 4. In all figures like Fig. 5, a complex $F$ is represented by its embedding $\Psi(F)$ (one image point per face).

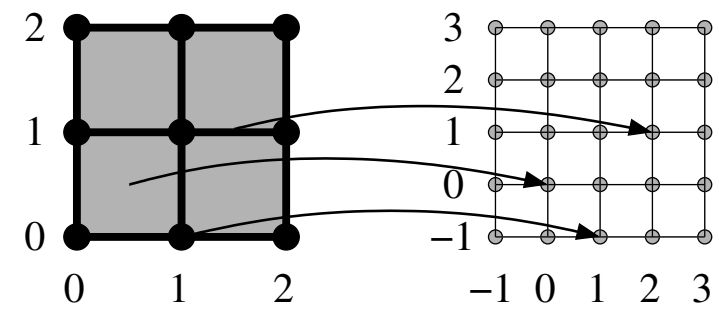

Figure 4. Illustration of the bijection $\Psi$ from $\mathbb{F}^{2}$ to $\mathbb{Z}^{2}$.

Depending on the parity of their coordinates, the elements of $\mathbb{Z}^{2}$ may represent different kinds of faces. The following classification will thus be useful in the sequel.

Definition 6. Let $i \in\{0,1,2\}$. We define the set $G_{i} \subset \mathbb{Z}^{2}$ as the set of elements in $\mathbb{Z}^{2}$ with exactly $i$ even coordinates, more precisely $G_{i}=\left\{\left(z_{1}, z_{2}\right) \in \mathbb{Z}^{2} \mid\left(\left(z_{1}+1\right) \bmod 2\right)+\right.$ $\left.\left(\left(z_{2}+1\right) \bmod 2\right)=i\right\}$.

From Def. 5 and Def. 6, we see that 2-faces are represented by points of $G_{2}$ (points with two even coordinates), 1 -faces by points of $G_{1}$, and 0 -faces by points of $G_{0}$.

Let us consider different approaches to define the Euclidean medial axis of a complex $F \preceq \mathbb{F}^{2}$.

The immediate approach consists of taking directly the Euclidean medial axis of the embedding of $F$ in $\mathbb{Z}^{2}$, that is, $\operatorname{MA}\left(\Psi(F), \mathbb{Z}^{2}\right)$. The result, however, is quite noisy and not useful in practice, as shows Fig. 5a. More precisely, the embedding of the complex $F$ is made of squares of $3 \times 3$ points, and thus its contour is "crenelated". This fact induces many medial axis points in the neighborhood of the object contour.

A second approach, that produces a better result, consists of taking the Euclidean medial axis of the embedding of $\mathrm{F}^{+}$ in $G_{2}$, that is, $M=\operatorname{MA}\left(\Psi\left(F^{+}\right), G_{2}\right)$. As an argument supporting this approach as well as the following one, let us observe that a complex is often obtained from a digital image by identifying each image pixel with a 2 -face [3]. In this particular case, $M$ would correspond to the Euclidean medial axis of the original image. The result is shown in Fig. 5b. The drawback of this approach is that it produces a rather "thick" medial axis.

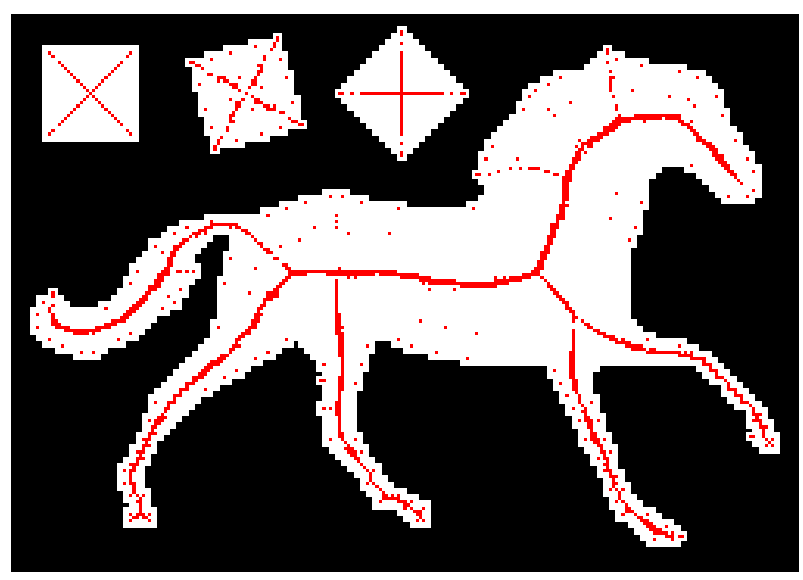

(a)

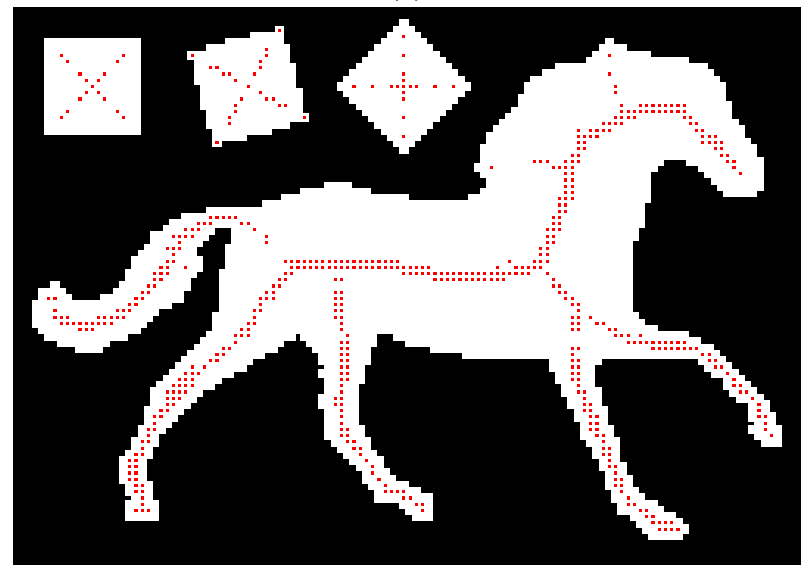

(b)

Figure 5. (a): A complex $F$ (in white), and the Euclidean medial axis of $\Psi(F)$ (highlighted). (b): A complex $F$ (in white), and the Euclidean medial axis of $\Psi\left(F^{+}\right)$in $G_{2}$ (highlighted).

We propose a third approach, that is to use the exact Euclidean medial axis in higher resolution (H-medial axis), introduced in [11].

Let us analyze further the second approach presented above. In Fig. 6, a detail of Fig. 5b is shown. In Fig. 6b, the points of $G_{2}$ are symbolized by small squares, and other points of $\mathbb{Z}^{2}$ by small discs. Note that $\Psi\left(F^{+}\right) \subset G_{2}$, and that $\operatorname{MA}\left(\Psi\left(F^{+}\right), G_{2}\right)$ is based on Euclidean balls in $G_{2}$ centered on points of $G_{2}$. For example, in Fig. 6(b), $B_{1}$ (squares surrounded by a dashed circle) is a maximal ball in $G_{2}$ centered on the point $X$ : it is strictly included in $B_{2}$, but $B_{2}$ is centered on $Y$ which is in $G_{1}$ and not in $G_{2}$. Thus, $X$ belongs to the medial axis.

The idea is to consider a larger number of Euclidean balls in $G_{2}$, by using the concept of $G_{2}$-balls. The $G_{2}$-balls are Euclidean balls in $G_{2}$, centered on any point of $\mathbb{Z}^{2}$, not nec- 


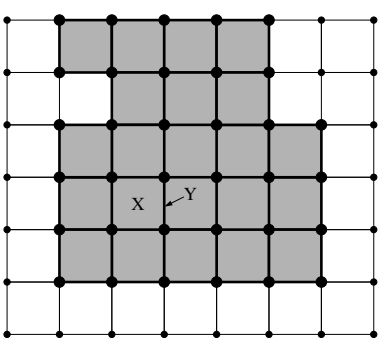

(a)

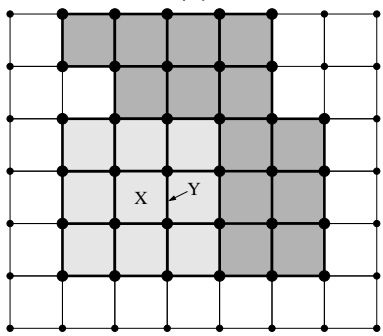

(c)

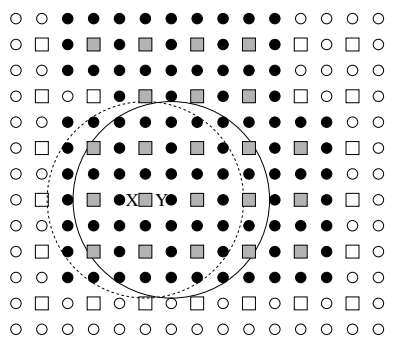

(b)

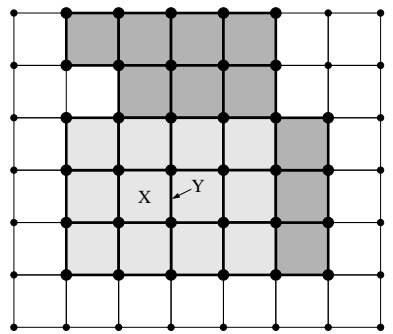

(d)
Figure 6. (a): A complex $F \preceq \mathbb{F}^{2}$, with $F^{+}$in gray. (b): The embedding $\Psi(F)$, with $\Psi\left(F^{+}\right)$in gray. The dashed circle helps to visualize the Euclidean ball $B_{1}$ in $G_{2}$, made of 9 points and centered on $X$, which is maximal for $\Psi\left(F^{+}\right)$, and the solid circle helps to visualize the $G_{2}$ ball $B_{2}$, made of 12 points and centered on $Y$, which is $G_{2}$-maximal for $\Psi(F)$. The ball $B_{1}$ is also a $G_{2}$-ball included in $\Psi\left(F^{+}\right)$, but not $G_{2^{-}}$ maximal. (c): A representation of $\Psi^{-1}\left(B_{1}\right)$ (in light gray). (d): A representation of $\Psi^{-1}\left(B_{2}\right)$ (in light gray).

essarily in $G_{2}$.

Definition 7. Let $x \in \mathbb{Z}^{2}, R \in \mathbb{N}$, the $G_{2}$-ball centered on $x$ with (squared) radius $R$ is the set $\left\{y \in G_{2} \mid d^{2}(x, y) \leq R\right\}$. Let $X \subset \mathbb{Z}^{2}$, we say that a $G_{2}$-ball $B$ is $G_{2}$-maximal for $X$ if $B$ is included in $X$ and maximal for this property, more precisely if $B \subseteq B^{\prime} \subseteq X$, with $B^{\prime}$ being a $G_{2}$-ball, implies $B=B^{\prime}$.

The family of $G_{2}$-balls includes all the Euclidean balls in $G_{2}$ considered by the previous approach, in addition to $G_{2}$-balls centered on points of $G_{1}$ and $G_{2}$-balls centered on points of $G_{0}$. For example, in Fig. $6 \mathrm{~b}, B_{2}$ is a $G_{2}$-ball (which is $G_{2}$-maximal). We can now give the definition of the $\mathrm{H}$-medial axis.

Definition 8. Let $X \subset \mathbb{Z}^{2}$. The $\mathrm{H}$-medial axis of $X$, denoted by $H M A(X)$, is the set of centers of all $G_{2}$-maximal $G_{2}$-balls for $X$.

Let $F \preceq \mathbb{F}^{2}$. The $\mathrm{H}$-medial axis of $F$, denoted by $\operatorname{HMA}(F)$, is defined by $H M A(F)=\Psi^{-1}(H M A(\Psi(F)))$.

An efficient algorithm for computing the $\mathrm{H}$-medial axis in $\mathbb{Z}^{2}$ and $\mathbb{Z}^{3}$, as well as a proof for this algorithm, has been proposed in [11]. The algorithm uses the same idea as the one presented in [10] for the exact Euclidean medial axis. It consists of two basic steps: i) calculate the exact Euclidean distance transform of $X$ (this can be done with a lineartime algorithm [9]) and ii) for each point of $X$, test if it is the center of a $G_{2}$-maximal $G_{2}$-ball. This test is done efficiently with the help of look-up tables. We invite the reader to consult the reference [11] for further details on the implementation of the $\mathrm{H}$-medial axis algorithm.

Let $F \in \mathbb{F}^{2}$. The H-medial axis $M=\operatorname{HMA}(F)$ is shown in Fig. 7a, and the corresponding subcomplex $M^{-}$ of $F$ is in Fig. 7b. Comparing Fig. 7a with Fig. 5b, one can observe that the $\mathrm{H}$-medial axis is "thinner" than the Euclidean medial axis of $\Psi\left(F^{+}\right)$. For a more precise evaluation of this statement, see [11]. Due to the space between points of the axis in Fig. 5b, this comparison will be more evident later, when we present the results of the homotopic skeletons constrained by these two axes (Fig. 8).

\section{New Euclidean homotopic skeleton algo- rithm}

We can now introduce our new algorithm. Before presenting its final version, we first introduce algorithms EHSa and EHSb which help to motivate and understand all the features of algorithm EuclideanHomotopicSkeleton presented hereafter.

Algorithm EHSa is the simplest one. It is essentially a parallel algorithm; notice, in particular, that no choice is made depending on the orientation of the object's contour.

Notice that all the values of the distance map $D_{H}^{2}$ are of the form $a^{2}+b^{2}$, where $a$ and $b$ are integers. In the following, $\mathbb{D}_{2}$ denotes the set of numbers which are the sum of two square integers.

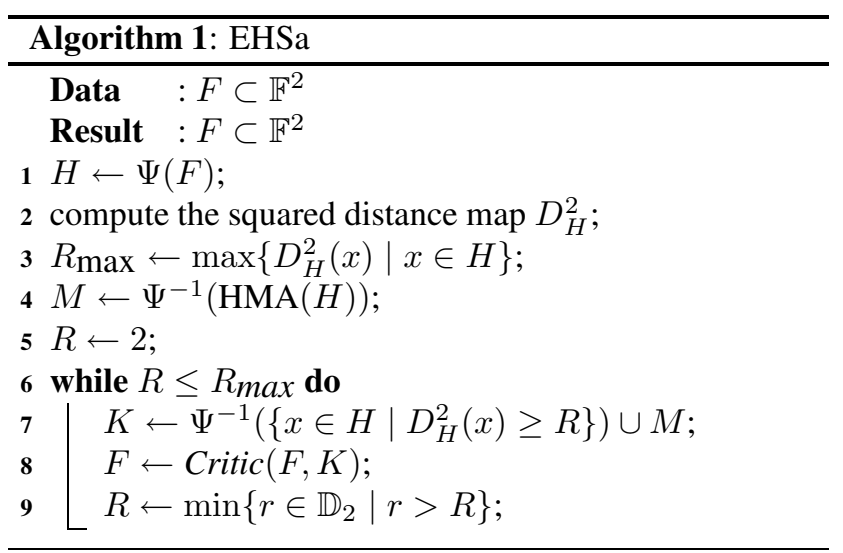

Notice that, in this algorithm, the constraint set $K$ needs not to be a complex. On the other hand, the set $F$ remains a complex during all the execution, by definition of $\operatorname{Critic}(F, K)$. 


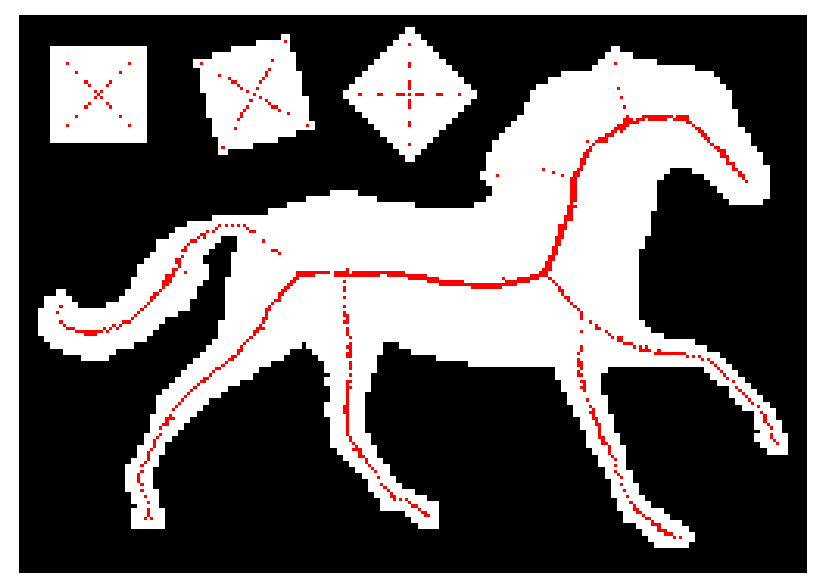

(a)

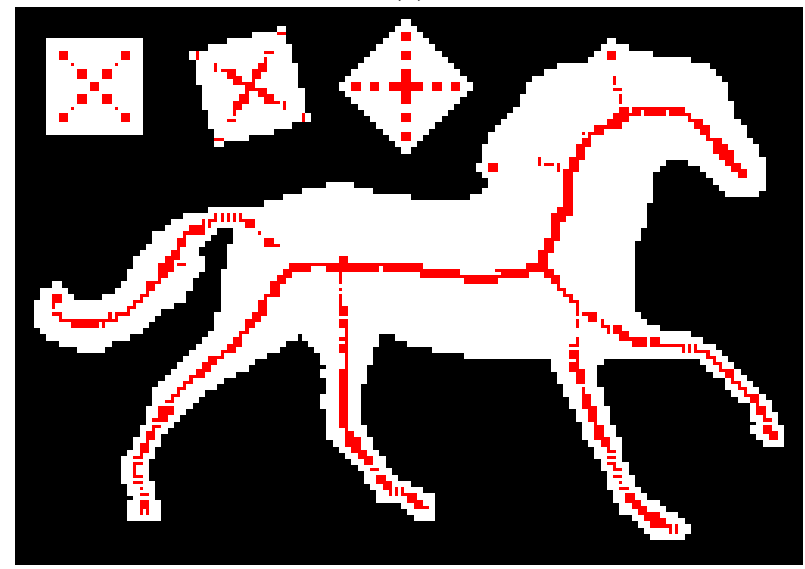

(b)

Figure 7. (a): The Euclidean H-medial axis of $F$ (highlighted). (b): The complex built from the Euclidean H-medial axis of $F$ (highlighted).

For an efficient sequential implementation, we introduce algorithm EHSb which can be implemented to run in $O(n \log n)$ time, where $n=|F|$, thanks to appropriate data structures (a balanced binary search tree for $Q$, chained lists for $X$ and $C$ ). The characterization of essential and critical faces is local and can be performed efficiently by using precomputed lookup tables.

Compare, in Fig. 8a and Fig. 8b, the relative thinness of our skeleton and the one obtained with the medial axis shown in Fig. 5b as constraint set (see also a detail in Fig. 9).

Algorithms EHSa and EHSb usually give satisfactory results on "small" images, such as the one of Fig. 8. However, especially for larger images, they do not guarantee that points of the homotopic skeleton outside the medial axis are "well centered" in the object. In other words, skeleton branches may fail to follow lines of steepest slope of the

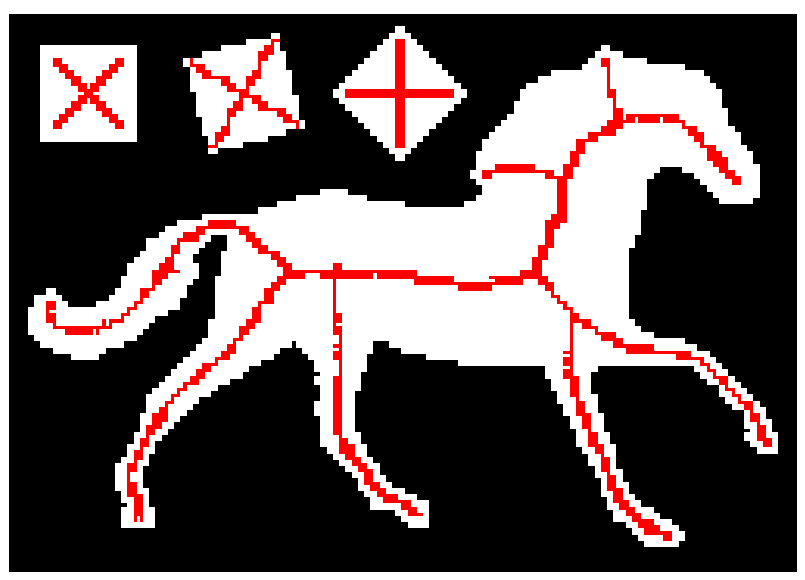

(a)

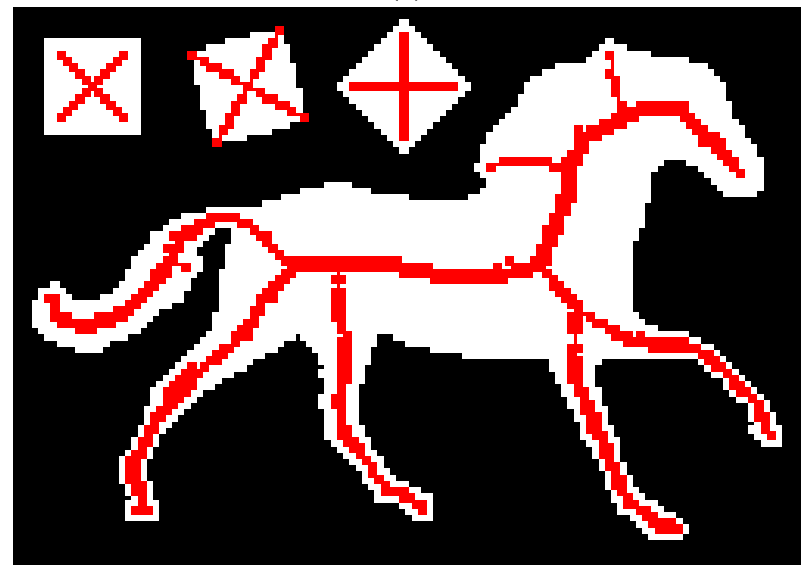

(b)

Figure 8. (a): Result of algorithm EHSb (or EHSa). (b): Result of a variant of algorithm EHSb taking as constraint set the medial axis of $\Psi\left(F^{+}\right)$shown in Fig. 5b.

Euclidean distance map.

This drawback has been well analyzed in [12] (see also [6]), for other thinning algorithms guided by Euclidean distance maps. In Fig. 10a, we give an illustration of the problem which may be encountered. A solution to this problem, which applies both for the 2D and 3D cases, has been proposed in [6] and can be adapted to the framework of critical kernels. It consists in defining a priority function which takes into account both the distance map and an auxiliary function defined in the neighborhood of each dynamically detected skeleton point.

Let $x \in \mathbb{Z}^{2}$, we define the neighborhood of $x$ as the set $\gamma(x)=\left\{y \in \mathbb{Z}^{2} \mid \max \left(\left|y_{1}-x_{1}\right|,\left|y_{2}-x_{2}\right|\right) \leq 1\right\}$. Let us define $\Gamma(x)=\gamma(x)$ if $x \in G_{0}$ or $x \in G_{1}$; and $\Gamma(x)=\gamma(\gamma(x))$ if $x \in G_{2}$. Let $x, y \in \mathbb{Z}^{2}$, we define $\delta(x, y)=D_{H}(x)+\left(D_{H}(y)-D_{H}(x)\right) / d(x, y)$. 


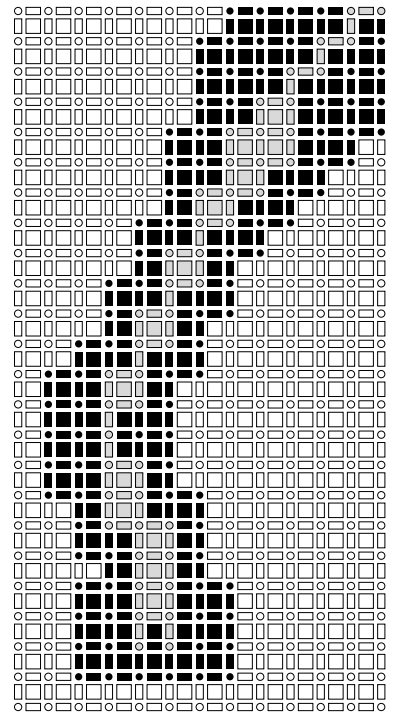

(a)

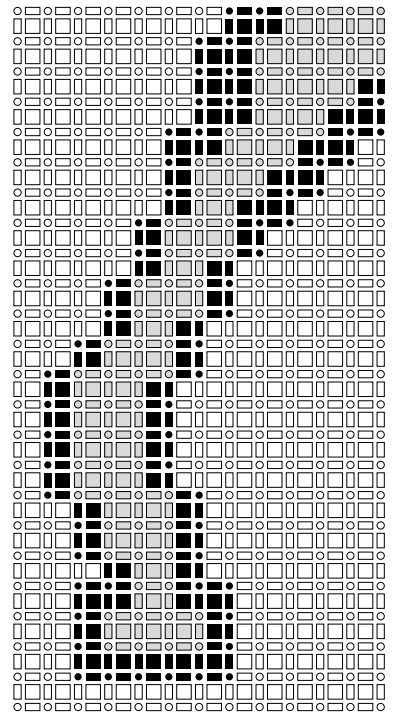

(b)
Figure 9. (a): Zoom on a part of Fig. 8a. (b): Zoom on a part of Fig. 8 b.

The justification of the expression of $\delta$ is the following. The quantity $\left(D_{H}(y)-D_{H}(x)\right) / d(x, y)$ represents the slope, in the distance map, between the point $x$ and the point $y$. For a given point $x$ detected as a skeleton point, we wish to classify the points of its neighborhood in ascending order of slope, rather than in ascending order of the distance map values, in order to guarantee that skeleton lines will follow the steepest slope.

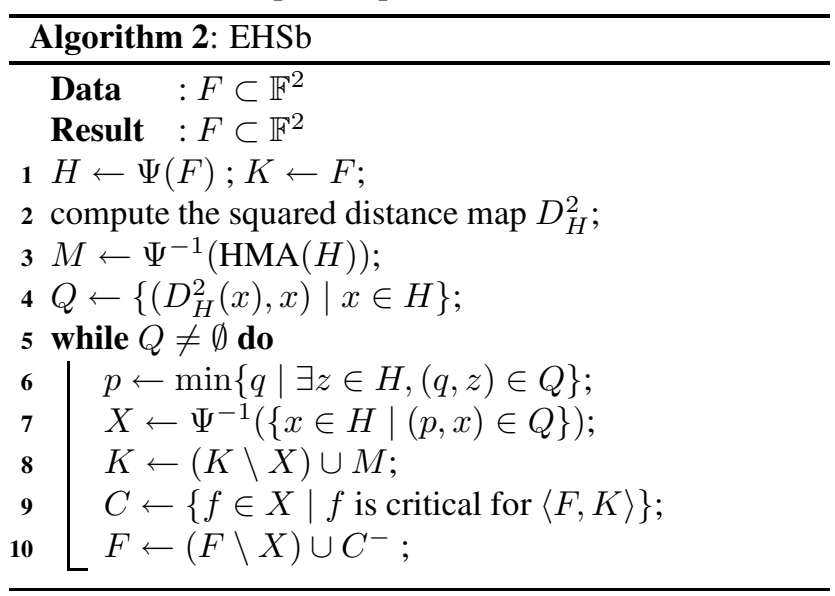

Compare the results of algorithms EHSb and EuclideanHomotopicSkeleton, for the detailed view of Fig. 10 and also for the larger image of Fig. 11 and Fig. 12.

\section{Conclusion}

Based on recent advances in the domains of parallel homotopic thinning and exact discrete Euclidean transforms, we proposed in this paper definitions and algorithms for the Euclidean homotopic skeleton based on critical kernels.

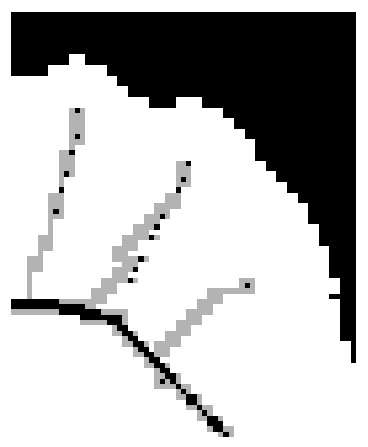

(a)

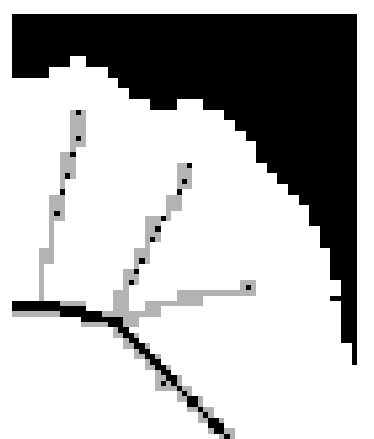

(b)
Figure 10. (a): Zoom on a part of a skeleton obtained by algorithm EHSb (the skeleton is in gray, and the H-medial axis is in black). (b): Zoom on a part of a skeleton obtained by algorithm EuclideanHomotopicSkeleton.

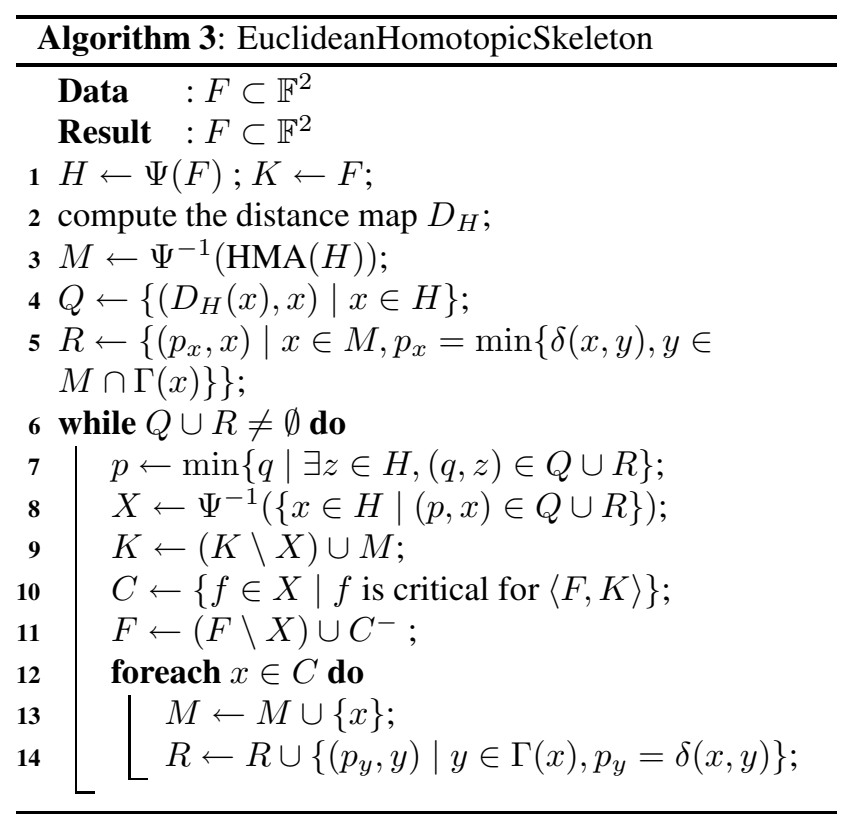

The framework of critical kernels allows to design efficient and topologically sound algorithms for parallel homotopic thinning without arbitrary decisions, leading to a unique result. The H-medial axis is a medial axis based on the Euclidean distance in a doubled resolution grid. After adapting the $\mathrm{H}$-medial axis to the framework of cubical complexes, we proposed a thinning algorithm which preserves the H-medial axis and follows the lines of steepest slope of the distance map. The skeleton obtained has the following characteristics: well-defined, homotopic, sufficient for the reconstruction of the original object, centered with respect to the (exact) Euclidean distance and thus weakly sensitive to rotations by arbitrary angles, and strictly invariant to rotations by multiples of $\pi / 2$. Furthermore it is thinner than the one obtained by a direct approach, it can be 


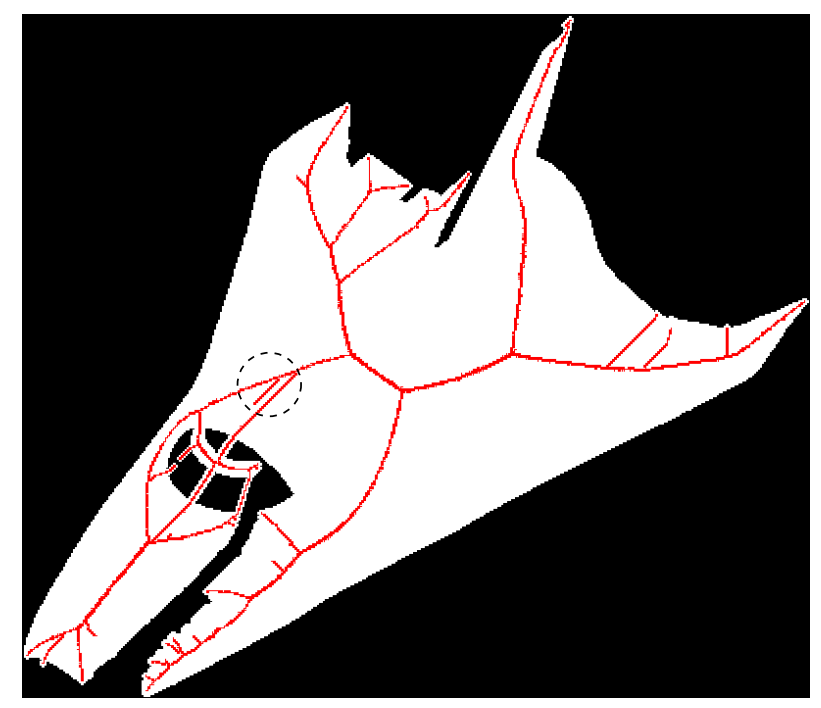

Figure 11. Skeleton obtained by algorithm EHSb. Notice the detail encircled by a dashed line, a branch of the skeleton does not correspond to any noticeable contour saliency.

computed efficiently, and it can be easily filtered by pruning the medial axis based on a radius and/or angle criterion (see $[12,6])$ in order to decrease sensitivity to small perturbations along the object shape. To our best knowledge, a skeleton with all these characteristics cannot be found in the literature.

\section{References}

[1] G. Bertrand. Skeletons in derived grids. In procs. Int. Conf. Patt. Recogn., pages 326-329, 1984.

[2] G. Bertrand. On critical kernels. Technical Report IGM2005-5, Institut Gaspard-Monge, Université de Marne-la-Vallée, France, 2005.

[3] G. Bertrand and M. Couprie. New 2d parallel thinning algorithms based on critical kernels. In Combinatorial Image Analysis, volume 4040 of LNCS, pages 45-59. Springer, 2006.

[4] H. Blum. An associative machine for dealing with the visual field and some of its biological implications'. Biological prototypes and synthetic systems, 1:244260, 1961.

[5] H. Blum. A transformation for extracting new descriptors of shape. In W. Wathendunn, editor, Models for the Perception of Speech and Visual Form, pages 362380. MIT Press, 1967.

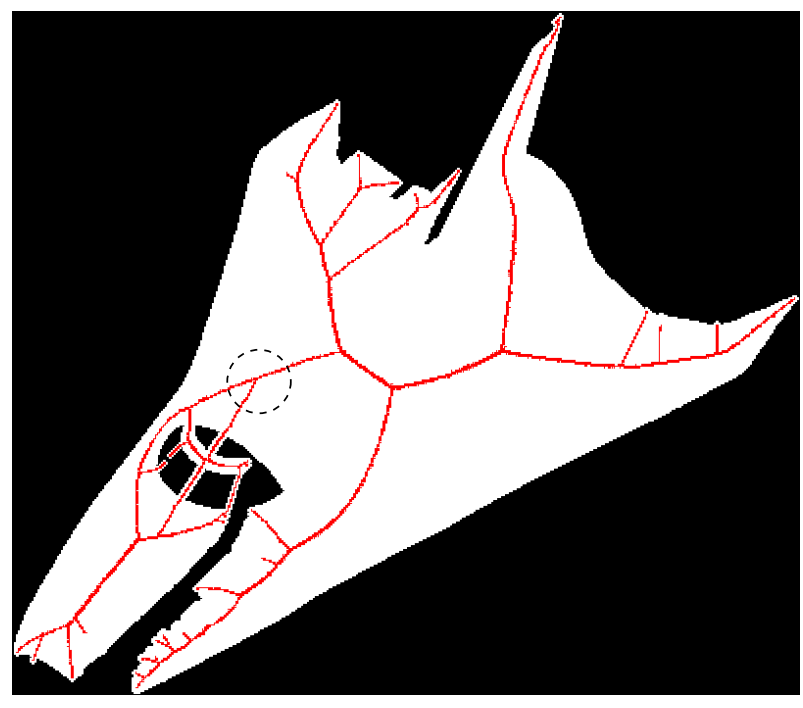

Figure 12. Skeleton obtained by algorithm EuclideanHomotopicSkeleton. Notice the difference in the encircled region.

[6] M. Couprie, D. Coeurjolly, and R. Zrour. Discrete bisector function and Euclidean skeleton in $2 \mathrm{~d}$ and $3 \mathrm{~d}$. Image and Vision Computing, 2006. accepted.

[7] E. Davies and A. Plummer. Thinning algorithms: a critique and a new methodology. Pattern Recognition, 14:53-63, 1981 .

[8] A. F. de Souza. Expansão por dilatação e por erosão visando a extração de esqueletos e contornos em imagens digitais. $\mathrm{PhD}$ thesis, INPE, Brazil, 2005.

[9] T. Hirata. A unified linear-time algorithm for computing distance maps. Information Processing Letters, 58(3):129-133, 1996.

[10] E. Rémy and E. Thiel. Exact medial axis with Euclidean distance. Image and Vision Computing, 23(2):167-175, 2005.

[11] A. V. Saúde, M. Couprie, and R. Lotufo. Exact Euclidean medial axis in higher resolution. In Discrete Geometry for Computer Imagery, volume 4245 of LNCS, pages 605-616. Springer, 2006.

[12] H. Talbot and L. Vincent. Euclidean skeletons and conditional bisectors. In Procs. VCIP'92, SPIE, volume 1818, pages 862-876, 1992.

[13] L. Vincent. Efficient computation of various types of skeletons. In Procs. Medical Imaging V, SPIE, volume 1445, pages 297-311, 1991. 